\title{
Caractérisation physico-chimique et diversité du peuplement phytoplanctonique des mares au sud de la réserve de faune de Togodo
}

(sud-Togo)

\author{
Djiedjom Lagno ASSOUGNON ${ }^{1 *}$, Hyppolite AGADJIHOUÈDÉ ${ }^{2}$, Kouami KOKOU ${ }^{1,3}$ et \\ Adédjobi Philippe LALÈYÈ ${ }^{4}$ \\ ${ }^{1}$ Laboratoire de Palynologie, Algologie et Paléoécologie (LaPAP), \\ Université de Lomé. BP : 1515 Lomé, Togo. \\ ${ }^{2}$ Ecole d'Aquaculture de la Vallée, Université Nationale d'Agriculture. BP : 43 Kétou, Bénin. \\ ${ }^{3}$ Laboratoire de Botanique et Ecologie Végétale, Faculté des Sciences, Université de Lomé, BP: 1515, Lomé, \\ ${ }^{4}$ Laboratoire d'Hydrobiologie et d'Aquaculture, Faculté des Sciences Agronomiques, \\ Université d'Abomey-Calavi, 01 BP : 526 Cotonou, Bénin. \\ *Auteur correspondant ; E-mail : lagnodjiedjom@gmail.com,Tel : +22891970714
}

\section{REMERCIEMENTS}

Ce travail a été financé par ARES dans le cadre des études du Master Régional en Monitoring des Ressources Aquatiques et Pêches Continentales de la Faculté des Sciences Agronomiques de l'Université d'Abomey-Calavi. Nous tenons à remercier aussi l'Union Economique Monétaire Ouest Africain (UEMOA) qui a financé ce travail à travers son Projet d'Appui à L'Enseignement Supérieur (PAES) dans les pays membres.

\section{RESUME}

Cette étude a été réalisée dans le but de déterminer les caractéristiques physico-chimiques et la diversité phytoplanctonique de l'eau des mares Dindin, Lotoè, Lagoè et Afi. Pour ce faire, dix sites de prélèvement ont été retenus et répartis dans ces mares dans le mois de juillet et août 2015. Les paramètres physico-chimiques ont été déterminés suivant les normes AFNOR. L'identification et le dénombrement du phytoplancton ont été faits au microscope photonique de marque Nikon Eclipse 0,90 Dry. L'étude a révélé une turbidité, un $\mathrm{pH}$ et un taux d'oxygène dissous élevés dans les mares Afi et Lagoè avec des valeurs maximales de 8,25 NTU; 8,14 et $9,75 \mathrm{mg} / \mathrm{L}$. A Dindin, une conductivité électrique élevée de $590 \mu \mathrm{S} / \mathrm{cm}$ et un taux d'oxydabilité au $\mathrm{KMnO}_{4}$ élevé étaient notés. La mare Lotoè est particulière par sa profondeur et sa transparence. La communauté phytoplanctonique des mares est composée de 107 espèces regroupées en 7 classes et 5 embranchements. Les espèces de la classe des Chlorophycées sont plus diversifiées. Au niveau des mares Afi et Lagoè, respectivement 62 et 65 espèces étaient recensées et à Dindin et Lotoè, respectivement 46 et 37 espèces. Cette étude montre en outre, une dominance d'Euglénophycées au niveau de la mare Dindin et de Cyanophycées au niveau des mares Afi et Lagoè. Le taux élevé d'oxygène dissous des mares Afi et Lagoè fait de ces mares des milieux eutrophes.

(C) 2017 International Formulae Group. All rights reserved.

Mots clés : Phytoplancton, Paramètres physico-chimiques, plan d'eau, Afrique de l'Ouest. 


\title{
Characterization of physico-chemical parameters and diversity of phytoplankton of some the ponds at the south side of the biosphere Park of Togodo (South Togo)
}

\begin{abstract}
This study was initiated in order to determine the physico-chemical parameters and the diversity of phytoplankton of the ponds Dindin, Lotoè, Lagoè and Afi. Then, covering all the ponds were selected in July and August 2015. The physico-chemical parameters were determined according to the AFNOR Standards. Identification and counts of phytoplankton were made in brand light microscope Nikon Eclipse 0.90 Dry. The study found a turbidity, $\mathrm{pH}$ and high oxygen levels in the ponds of Afi and Lagoè with respective maximum values are $8.25 \mathrm{NTU} ; 8.14$ and $9.75 \mathrm{mg} / \mathrm{L}$. At Dindin, a high electrical conductivity of about $590 \mu \mathrm{S} / \mathrm{cm}$ and high oxydability of $\mathrm{KMnO} 4$ were observed. Lotoè, was particular by its depth and high transparency. According to the diversity of phytoplankton population, 107 species grouped into 7 classes and 5 phyla were identified. The species of the class of Chlorophyceae were more diversified. At Afi and Lagoè ponds 62 and 65 species were respectively identified. But at Dindin and Lotoè, 46 and 37 species were respectively identified. This study also shows a dominance of Euglenophyceae with a high component at the Dindin pond and Cyanophyceae at Afi and Lagoè ponds. The high dissolved oxygen content of the Afi and Lagoè pools makes these pools eutrophic pools.
\end{abstract}

(C) 2017 International Formulae Group. All rights reserved.

Keywords: Phytoplankton, physico-chemical parameters, pond, West Africa.

\section{INTRODUCTION}

Le réseau hydrographique du Togo est composé d'un nombre important d'écosystèmes d'eau douce d'intérêt socioéconomique capital dont la qualité ne cesse de se dégrader de jour en jour à cause de la forte pression anthropique (Atanle et al., 2013).

Dans ces écosystèmes aquatiques, le phytoplancton constitue la base de la chaîne trophique (Azam et Malfatti, 2007). Il peut former des efflorescences suite à la prolifération d'une ou de quelques espèces dans des conditions hydroclimatiques favorables; ce qui peut entrainer des conséquences sanitaires, écologiques et économiques. Toutefois, au Togo, peu d'études ont été consacrées à ces écosystèmes. Pour le phytoplancton et la pollution de l'eau, quelques études ont été réalisées (Edorh et al., 2008 ; Atanle et al., 2013 ; Issifou et al., 2014).

Parmi les plans d'eau du Togo, se trouvent des mares au sud de la Réserve de
Faune de Togodo au Sud-Togo. En effet, ces mares ne dépendent pas seulement du fonctionnement de leurs composantes internes, mais aussi des contraintes locales; apports de leurs bassins versants. En outre, elles sont entourées par des agglomérations avec les différentes activités humaines telles que: l'agriculture avec l'utilisation des engrais et pesticides. L'agriculture contribue à l'enrichissement des mares en nutriments (phosphores, nitrates, etc.). Or une augmentation continue en quantité de ces nutriments est un facteur principal d'eutrophisation des plans d'eau (Jiang et Shen, 2006). Par ailleurs, leur utilisation occasionne aussi la prolifération des plantes aquatiques et des algues dont certaines sont indicatrices du niveau de pollution des eaux (MERF, 2002). Quant aux déchets ménagers, pâturage avec les excréments d'animaux et le lessivage des terres environnantes; ils enrichissent ces eaux en matières organiques. Il est à noter aussi que ces mares, alimentées 
par le fleuve Mono en période de crue sont en contact avec les déchets industriels qui contiennent du phosphate, des métaux lourds et beaucoup de sels dissouts. A l'instar des écosystèmes d'eau douce pollués, ces mares sont en train de perdre beaucoup d'atouts. Ce sont des mares riches en produits halieutiques et qui jouent un rôle important dans la conservation de la biodiversité (diverses espèces de poissons, hippopotames, crocodiles, etc.). Aujourd'hui, ces mares sont envahies par des espèces végétales, avec des bordures complètement transformées en roselières et marécages. L'appauvrissement du milieu dû à la dégradation de la qualité de l'eau des mares entraine de jour en jour un exode rural massif et des problèmes de santé. La connaissance de l'état de pollution de ces eaux du lac peut alors amener à trouver des approches de solution. Face à cette situation, cette étude a été initiée pour déterminer les caractéristiques physico-chimiques et la diversité phytoplanctonique indicatrice de la qualité de l'eau des mares.

\section{MATERIEL ET METHODES}

\section{Cadre d'étude et sites d'échantillonnages}

Localisées dans la Région Maritime au Togo, les mares Dindin, Lotoè, Afi et Lagoè (Photographie 1,2,3,4) sont situées au sud de la réserve de Faune de Togodo au Sud-Togo plus précisément dans la préfecture de Yoto (Figure 1). Avec des superficies moyennes respectives de 29,11 ha; 37,55 ha; 128,74 ha et de 29,61 ha, ces plans d'eau sont d'une grande importance socio-économique tant pour le pays que pour les populations autochtones. Elles sont influencées en période de crue par le fleuve Mono. Le bassin versant de ces mares, à l'instar du sud-Togo, jouit d'un climat de type équatorial guinéen à deux saisons de pluies alternant avec deux saisons sèches. Les principales activités économiques relevées dans les agglomérations sont la pêche, l'agriculture et/ou l'élevage.

Les données de ce travail proviennent de dix (10) sites d'échantillonnages choisis de façon à représenter le plan d'eau sur sa superficie. Deux (2) sites sont choisis sur chacune des mares (Dindin, Lotoè et Lagoè) et quatre (4) sur la mare Afi à cause de sa superficie qui fait plus de deux fois celle des autres (Figure 1).

\section{Prélèvements de l'eau et détermination des paramètres physico-chimiques}

Les prélèvements d'eau ont été effectués en juillet et août 2015. Les prélèvements ont été faits en surface et en profondeur. Pour les analyses physicochimiques, des échantillons d'eau ont été prélevés dans des bouteilles plastiques de 1,5 litre. In situ, la conductivité, la température, le $\mathrm{pH}$, l'oxygène dissous, la transparence et la profondeur ont été mesurés. Les autres paramètres physico-chimiques ont été déterminés au laboratoire. Pour les analyses, les méthodes AFNOR (1997) ont été utilisées (Tableau 1).

La caractérisation biologique a été réalisée à travers une analyse qualitative de la flore algale. Les prélèvements ont été effectués dans des piluliers de $15 \mathrm{ml}$. Le phytoplancton a été immédiatement fixé au lugol, gardé au frais et à l'obscurité jusqu'au laboratoire. Chaque échantillon est laissé au repos pendant 24 à 48 heures au minimum pour permettre la sédimentation des algues. L'eau a été ensuite prélevée au-dessus du résidu algal par siphonage. La solution résiduelle concentrée en phytoplancton a été conservée dans le pilulier. Un volume précis $(50 \mu \mathrm{l})$ de chaque échantillon homogénéisé a été monté entre lame et lamelle puis observé à l'aide d'un microscope photonique de marque Nikon Eclipse 0,90 Dry aux objectifs 10X et 40X. Pour chaque échantillon, trois lames ont été préparées afin de s'assurer de la reproductibilité des lames. Les observations ont été faites suivant des transects verticaux. Selon la morphologie générale des taxons observés, une détermination des espèces a été faite à partir de la photothèque de référence des algues du Togo (Issifou et al., 2014) et des 
travaux de Bourrelly (1968, 1970, 1972), Komarek et Anagnostidis (1999), Lavoie et al. (2008). Parallèlement à l'identification des espèces, elles sont également comptées. Les données issues du comptage ont servi à la détermination des densités de ces organismes phytoplanctoniques.

\section{Analyse statistique}

Les valeurs de la plupart des paramètres mesurés lors de cette étude ont été moyennées pour chaque site. L'ensemble des résultats a été traité dans un premier temps avec le Tableur Excel (2010). Le test de Kruskal-Wallis ainsi que le t-test ont été utilisés pour évaluer les variations des différents paramètres physico-chimiques des plans d'eau et l'évolution de l'abondance du phytoplancton au cours des mois de l'étude. L'analyse descriptive a été faite pour calculer le coefficient de variation des différents paramètres à l'aide du logiciel Minitab.
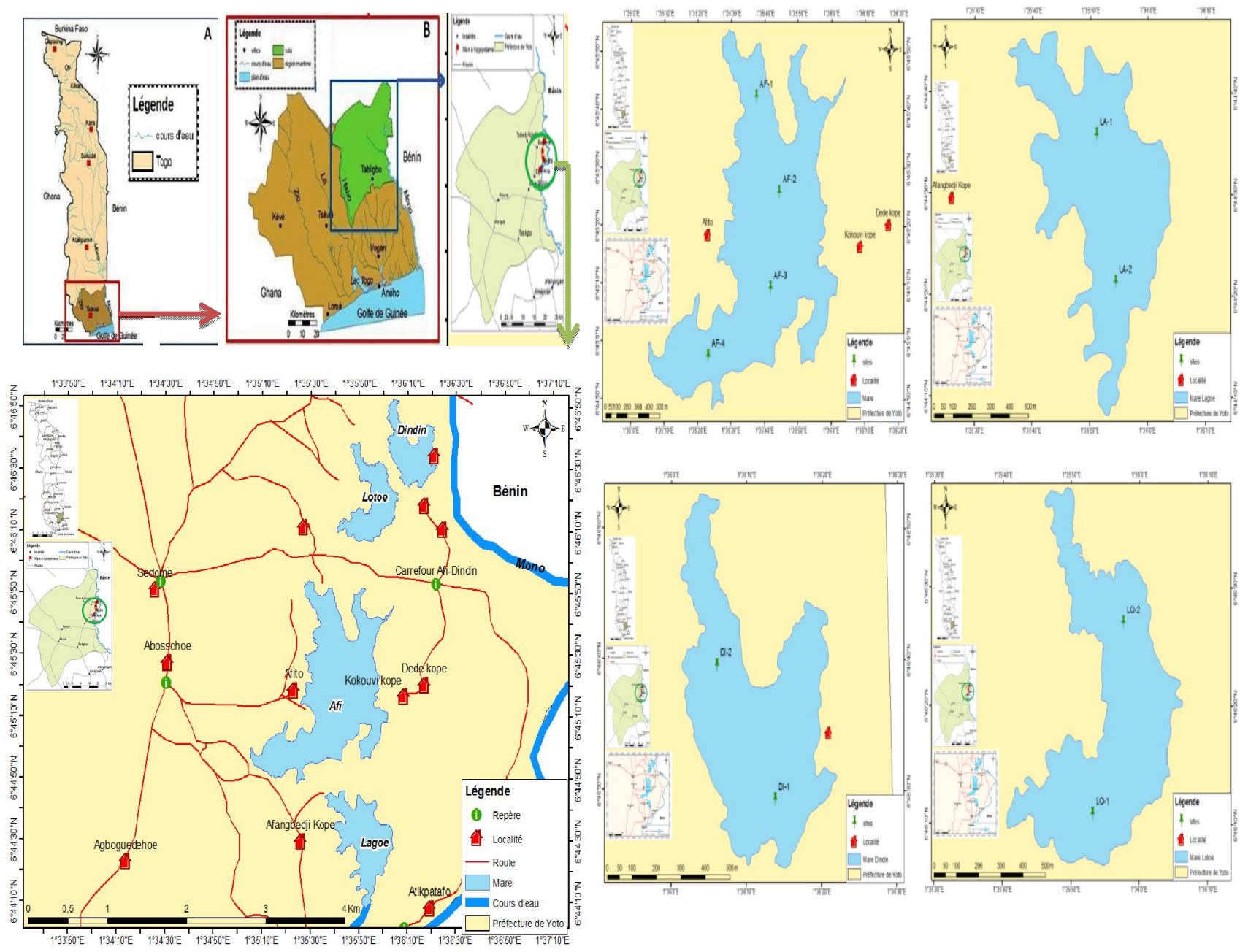

Figure 1: Carte du Togo mettant en relief la Région Maritime (A), la préfecture de Yoto (B), la localisation du milieu d'étude (les mares Afi, Lagoè, Lotoè et Dindin) (C) et la localisation des sites d'échantillonnages dans chacune des mares (Image Landsat, 2015). 
Tableau 1: Méthodes de détermination des paramètres physico-chimiques de l'eau.

\begin{tabular}{|c|c|c|c|c|}
\hline Paramètres & Méthodes & Norme AFNOR & Précision & Matériel utilisé \\
\hline $\begin{array}{l}\text {-Conductivité, } \\
\text {-Température, } \\
\text { - pH }\end{array}$ & $\begin{array}{l}\text {-Conductimétrie } \\
\text { - Electrométrie } \\
\text { - Electrométrie }\end{array}$ & $\begin{array}{l}\text {-NFT 90-031 } \\
\text {-NFT 90-100 } \\
\text {-NFT 90-008 }\end{array}$ & $\begin{array}{c} \pm 0.01 \mathrm{NTU} \\
\pm 0,01{ }^{\circ} \mathrm{C} \\
\pm 0.01\end{array}$ & Multimètre WTW Multi 342 \\
\hline Oxygène dissous & - & - & - & Oxymètre WTW Oxi 340 \\
\hline Transparence & Secchi & NF EN ISO 7027 & - & Oxymètre WTW Oxi 340 \\
\hline Profondeur & Corde lestée graduée & & - & Corde graduée et lestée \\
\hline Turbidité & Néphélométrie & NFT 90-33 & $\pm 0.01 \mathrm{NTU}$ & $\begin{array}{c}\text { Turbidimètre DRT100B } \\
\text { model } 20012\end{array}$ \\
\hline $\begin{array}{l}\text {-Ammonium }\left(\mathrm{NH}_{4}^{+}\right) \\
\text {-Nitrate }\left(\mathrm{NO}_{3}^{-}\right) \\
\text {-Orthophosphate }\left(\mathrm{PO}_{4}{ }^{3-}\right) \\
\text {-Nitrite }\left(\mathrm{NO}_{2}^{-}\right)\end{array}$ & Spectrophotométrie & $\begin{array}{l}\text { - NFT 90-015 } \\
\text { - NFT 90-013 } \\
\text { - NFT 90-013 } \\
\text { - NFT 90-012 }\end{array}$ & 1 à $2 \%$ & $\begin{array}{c}\text { Spectromètre } \\
\text { Digitron Elvi } 675\end{array}$ \\
\hline Oxydabilité au $\mathrm{KMnO}_{4}$ & Basique/Chaud & EN ISO 8467 & $\pm 0,5 \mathrm{mg} / \mathrm{L}$ & Verrerie de laboratoire \\
\hline
\end{tabular}

\section{RESULTATS}

Caractéristiques physico-chimiques des mares

Température, conductivité électrique et pH de l'eau

En juillet, les températures enregistrées étaient plus élevées que celles d'août. Les moyennes étaient de $28,6{ }^{\circ} \mathrm{C} ; 28,3{ }^{\circ} \mathrm{C}$ et 27,8 ${ }^{\circ} \mathrm{C}$ respectivement au niveau des mares Dindin, Lotoè et Lagoè. Au niveau de la mare Afi, elle était de $27,5{ }^{\circ} \mathrm{C}$. En août, la température moyenne était de $27,3^{\circ} \mathrm{C}$ pour les mares Dindin et Lotoè et de $27,6{ }^{\circ} \mathrm{C}$ pour Lagoè et Afi. Au niveau des mares, les variations mensuelles sont faibles (ANOVA, Kruskal-Wallis, $p>0,05 ;$ T-Test, $p>0,05$ ) (Tableau 2).

La conductivité la plus élevée était obtenue en août et la plus faible en juillet. La mare Dindin présentait les conductivités les plus élevées et Lotoè les plus faibles (Tableau 2).

Pour ce qui est du $\mathrm{pH}$, les mares Dindin et Lotoè présentaient des $\mathrm{pH}$ voisins de la neutralité, Lagoè et Afi des $\mathrm{pH}$ basiques. Aucune différence significative n'est notée entre les mois (ANOVA, Kruskal-Wallis, $p>$ 0,$05 ;$ T-Test, $p>0$,05) (Tableau 2).

Turbidité, oxygène dissous, oxydabilité au $\mathrm{KMnO}_{4}$

La moyenne de la turbidité la plus élevée était obtenue en août dans la mare Afi $(8,63 \mathrm{NTU})$. Pour ce qui concerne la teneur en oxygène dissous dans les mares, la moyenne la plus élevée était obtenue dans la mare Afi en juillet et la plus faible dans la mare Dindin en août. En ce qui concerne l'oxydabilité au $\mathrm{KMnO}_{4}$, la moyenne la plus élevée est obtenue dans la mare Dindin $\left(6,45 \mathrm{mgO}_{2} / \mathrm{L}\right)$ en août et la plus faible dans la mare de Lotoè $(3 \mathrm{mg} / \mathrm{L})$ en juillet. Les variations mensuelles de ces différents paramètres sont non négligeables, mais pas significatives au niveau des mares (ANOVA, Kruskal-Wallis, $p>$ 0,$05 ;$ T-Test, $p>0,05$ ) (Tableau 3).

\section{Minéraux azotés et l'orthophosphate}

Les teneurs en ions nitrites $\left(\mathrm{NO}_{2}{ }^{-}\right)$ étaient inférieures à $0,025 \mathrm{mg} / \mathrm{L}$ et celles des ions nitrates $\left(\mathrm{NO}_{3}^{-}\right)$à $0,5 \mathrm{mg} / \mathrm{L}$ sauf au niveau du site $\mathrm{AF}_{4}$ où elle était égale à $0,53 \mathrm{mg} / \mathrm{L}$ en août. Quant aux teneurs en ammonium $\left(\mathrm{NH}_{4}{ }^{+}\right)$, elles étaient inférieures à $0,05 \mathrm{mg} / \mathrm{L}$ en juillet mais avec des valeurs supérieures à 
0,05 mg/L en août (Tableau 4). S'agissant de l'orthophosphate, dans les mares leurs valeurs étaient toutes inférieures à $0,2 \mathrm{mg} / \mathrm{L}$ tout au long de cette étude (Tableau 4).

\section{Profondeur et transparence}

Les profondeurs moyennes ont varié entre $0,65 \mathrm{~m}$ obtenue dans la mare Dindin et $1,28 \mathrm{~m}$ dans la mare Lagoè. Quant aux profondeurs moyennes du plan d'eau, la valeur la plus élevée $(2,90 \mathrm{~m})$ était obtenue en juillet et la plus faible $(1,9 \mathrm{~m})$ en août. La mare Lagoè reste la mare la plus profonde. La mare Lagoè avait la transparence la plus élevée $(128 \mathrm{~cm})$. Les variations mensuelles sont faibles (ANOVA, Kruskal-Wallis, $p>$ 0,05; T-Test, $p>0,05$ ) (Tableau 5).

\section{Etat de la biodiversité phytoplanctonique}

Un total de cent sept (107) espèces regroupées en 7 classes et 5 embranchements ont été recensés dans les quatre mares. Il s'agit de la classe des Cyanophycées (embranchement des Cyanophytes), des Euglénophycées (embranchement des Euglénophytes), des Conjugatophycées, des Chlorophycées (embranchement des Chlorophytes), des Cryptophycées (embranchement des Pyrrhophytes), des Xanthophycées, des Diatomophycées (embranchement des Chromophytes). La classe des Chlorophycées est la classe la plus diversifiée avec $33 \%$ du peuplement phytoplanctonique (Figure 2). Certaines de ces espèces sont représentées sur la planche 1 .

A Dindin, 26 espèces ont été recensées en juillet. Elles sont réparties dans 7 classes. La classe des Chlorophycées (27\%) suivie de celle des Euglénophycées (23\%) sont les plus diversifiées. En août, le nombre d'espèces est passé à 32 réparties dans 6 classes. Au cours de ce mois, la classe la plus diversifiée était celle des Euglénophycées (38\%) suivie de celle des Chlorophycées (28\%). La classe des Cryptophycées restait la moins diversifiée au cours des deux (2) mois d'étude. Parlant de l'abondance, les Euglenophycées étaient les plus abondantes $\left(50.10^{3} \mathrm{ind} / \mathrm{L}\right.$ en juillet et $91.10^{3} \mathrm{ind} / \mathrm{L}$ en août) avec une densité relative de $72,96 \%$ et $69,44 \%$. Elles sont suivies des Chlorophycées durant les mois de juillet et août. Les Xanthophycées étaient les moins abondantes durant les deux mois. La densité absolue des Xanthophycées était de $1.10^{3}$ ind/L en juillet et absente en août (Tableau 6).

Dans la mare Lotoè, 23 et 28 espèces appartenant à 6 classes étaient respectivement recensées en juillet et août. La classe des Cyanophycées était la plus diversifiée (31\%) en juillet alors qu'en août, la classe des Euglénophycées était la plus diversifiée (50\%). Toutes les classes d'algues sauf les Euglénophycées et les Cryptophycées avaient eu leur forte densité en juillet. La dominance dans cette mare était assurée par les Cyanophycées $\left(21.10^{3} \mathrm{ind} / \mathrm{L}\right)$ avec une densité relative de $37 \%$ en juillet suivie des Chlorophycées $\left(13.10^{3} \mathrm{ind} / \mathrm{L}\right)$ avec une densité relative de $23,25 \%$. En août, les Euglénophycées $\left(63.10^{3} \mathrm{ind} / \mathrm{L}\right)$ avec une densité relative de $67,83 \%$ étaient les plus abondantes suivies des Cryptophycées $\left(50.10^{3}\right.$ ind/L; 8,70\%). Lors de cette étude, les Conjugatophycées étaient les moins abondantes $\left(2.10^{3} \mathrm{ind} / \mathrm{L}\right)$ (Tableau 6).

Les espèces recensées à Lagoè étaient au nombre de 43 en juillet avec la classe des Cyanophycées comme la plus diversifiée $(30 \%)$. En août, le nombre d'espèces recensées était de 55. La classe des Chlorophycées était la plus diversifiée avec $29 \%$ du peuplement phytoplanctonique. $\mathrm{Au}$ cours des deux mois d'étude, la dominance du phytoplancton était assurée par les Cyanophycées (respectivement $1543.10^{3}$ ind/ $\mathrm{L}$ en juillet et $516.10^{3} \mathrm{ind} / \mathrm{L}$ en août) avec une densité relative de $90,70 \%$ en juillet et $74,44 \%$ en août. La classe la moins dominante était la classe des Conjugatophycées avec une densité relative de $0,44 \%$ en juillet et Cryptophycées en août (densité relative de 0,38\%) (Tableau 6).

Quant à Afi, en juillet, 36 espèces appartenant à 5 classes étaient inventoriées. 
Les classes des Cyanophycées et des Chlororphycées y prédominaient avec un taux de $39 \%$ chacune. La classe des Conjugatophycées était la moins diversifiée avec 5\%. En août, le nombre d'espèces était passé à 48 avec une prédominance de la classe des Chlorophycées (38\%) suivie de celle des Cyanophycées (31\%). Les classes des Conjugatophycées et Diatomophycées étaient les moins représentées avec $8 \%$ du peuplement phytoplanctonique. La dominance était aussi assurée par les Cyanophycées avec respectivement $3229.10^{3}$ ind/L en juillet et 3 $355.10^{3}$ ind/L en août soit $86,88 \%$ en juillet et $83,90 \%$ en août. Elle était suivie des Chlorophycées au cours de l'étude avec la classe des Euglénophycées la moins représentée en juillet et la classe des Diatomophycées en août. Le mois d'août était caractérisé par une plus forte densité des classes phytoplanctoniques recensées sauf chez les Chlorophycées et Diatomophycées (Tableau 7).

Tableau 2:Température, conductivité électrique, $\mathrm{pH}$ mesurés dans les mares.

\begin{tabular}{|c|c|c|c|c|c|c|c|}
\hline \multirow[t]{2}{*}{ Mares } & \multirow[t]{2}{*}{ Paramètres } & \multicolumn{2}{|c|}{ Température $\left({ }^{\circ} \mathrm{C}\right)$} & \multicolumn{2}{|c|}{ Conductivité $(\mu \mathrm{S} / \mathrm{cm})$} & \multicolumn{2}{|c|}{ pH } \\
\hline & & juillet & août & juillet & août & juillet & août \\
\hline \multirow{4}{*}{ Dindin } & DI-1 & 28,8 & 27,6 & 585 & 587 & 6,85 & 7,2 \\
\hline & DI-2 & 28,5 & 27 & 590 & 593 & 6,84 & 7 \\
\hline & Moy & 28,65 & 27,3 & $\mathbf{5 8 7 , 5}$ & 590 & 6,845 & 7,1 \\
\hline & CV $(\%)$ & 0,74 & 1,55 & 0,6 & 0,72 & $\mathbf{0 , 1}$ & 1,99 \\
\hline \multirow{4}{*}{ Lotoè } & LO-1 & 28,7 & 27,2 & 384 & 391 & 7,01 & 7,19 \\
\hline & LO-2 & 27,9 & 27,4 & 393 & 394 & 7,36 & 7,52 \\
\hline & Moy & 28,3 & 27,3 & 388,5 & 392,5 & 7,185 & 7,355 \\
\hline & CV $(\%)$ & 2 & $\mathbf{0 , 5 2}$ & 1,64 & 0,54 & 3,44 & $\mathbf{3 , 1 7}$ \\
\hline \multirow{4}{*}{ Lagoè } & LA-1 & 27,8 & 27,4 & 404 & 414 & 7,78 & 7,69 \\
\hline & LA-2 & 27,9 & 27,8 & 404 & 414 & 7,66 & 7,62 \\
\hline & Moy & 27,85 & 27,6 & 404 & 414 & 7,72 & 7,655 \\
\hline & CV (\%) & 0,25 & 1,02 & $\mathbf{0}$ & $\mathbf{0}$ & 1,1 & 0,65 \\
\hline \multirow{6}{*}{ Afi } & AF-1 & 27,4 & 27,5 & 409 & 418 & 8,08 & 7,97 \\
\hline & AF-2 & 27,4 & 27,7 & 409 & 417 & 8,23 & 8,06 \\
\hline & AF-3 & 27,4 & 27,6 & 408 & 416 & 8,21 & 8,02 \\
\hline & AF-4 & 27,8 & 27,6 & 408 & 415 & 8,14 & 7,95 \\
\hline & Moy & 27,5 & 27,6 & 408,5 & 416,5 & 8,165 & 8 \\
\hline & CV (\%) & 0,73 & 0,3 & 0,14 & 0,31 & 8,08 & 7,95 \\
\hline
\end{tabular}

DI-1 : Dindin site 1, DI-2 : Dindin site 2, LO-1 : Lotoè site 1, LO-2 : Lotoè site 2, LA-1 : Lagoè site 1, LA-2 : Lagoè site 2, AF-1 : Afi site 1, AF-2 : Afi site 2, AF-3 : Afi site 3, AF-4 : Afi site 4, Moy : Moyenne ; CV : Coefficient de Variation. 
Tableau 3: Turbidité, oxygène dissous, oxydabilité au $\mathrm{KMnO}_{4}$ mesurés dans les mares.

\begin{tabular}{|c|c|c|c|c|c|c|c|}
\hline \multirow[t]{2}{*}{ Mares } & \multirow[t]{2}{*}{ Paramètres } & \multicolumn{2}{|c|}{ Turbidité (NTU) } & \multicolumn{2}{|c|}{ Oxygène dissous (mg/L) } & \multicolumn{2}{|c|}{$\mathrm{KMnO}_{4}\left(\mathrm{mgO}_{2} / \mathrm{L}\right)$} \\
\hline & & juillet & août & juillet & août & juillet & août \\
\hline \multirow{5}{*}{ Dindin } & DI-1 & 1,5 & 1,5 & 6,9 & 6,2 & 5,7 & 6,9 \\
\hline & DI-2 & 1,3 & 4,8 & 6,7 & 3,4 & 5,9 & 6 \\
\hline & Moy & 1,4 & 3,15 & 6,8 & 4,8 & 5,8 & 6,45 \\
\hline & CV (\%) & 10,1 & 74,08 & 2,08 & 41,25 & 2,44 & 9,87 \\
\hline & LO-1 & 1,1 & 1,6 & 7,4 & 3,9 & 3,1 & 3,3 \\
\hline \multirow[t]{4}{*}{ Lotoè } & LO-2 & 1,3 & 1,2 & 7,6 & 5,4 & 2,9 & 3,3 \\
\hline & Moy & 1,2 & 1,4 & 7,5 & 4,65 & 3 & 3,3 \\
\hline & CV $(\%)$ & 11,79 & 20,2 & 1,89 & 22,81 & 4,71 & $\mathbf{0}$ \\
\hline & LA-1 & 2,2 & 3,2 & 9,8 & 7,4 & 3,5 & 5,2 \\
\hline \multirow[t]{6}{*}{ Lagoè } & LA-2 & 2,4 & 4,6 & 9,6 & 7,7 & 4,3 & 3,2 \\
\hline & Мoy & 2,3 & 3,9 & 9,7 & 7,55 & 3,9 & 4,2 \\
\hline & CV (\%) & 6,15 & 25,38 & 1,46 & 2,81 & 14,5 & 33,67 \\
\hline & AF-1 & 5,5 & 10,9 & 9,4 & 8,7 & 3,1 & 5,2 \\
\hline & AF-2 & 5,7 & 10,8 & 9,7 & 9,1 & 2,9 & 7,4 \\
\hline & AF-3 & 5,3 & 9,6 & 10,5 & 9 & 4,2 & 7,2 \\
\hline \multirow[t]{3}{*}{ Afi } & AF-4 & 5,7 & 3,2 & 10,2 & 9 & 4,5 & 4,9 \\
\hline & Мoy & 5,55 & 8,63 & 9,95 & 8,95 & 3,675 & 6,175 \\
\hline & CV $(\%)$ & 3,45 & 42,49 & 4,96 & 1,94 & 21,58 & 21,17 \\
\hline
\end{tabular}

DI-1 : Dindin site 1, DI-2 : Dindin site 2, LO-1 : Lotoè site 1, LO-2 : Lotoè site 2, LA-1 : Lagoè site 1, LA-2 : Lagoè site 2, AF-1 Afi site 1, AF-2 : Afi site 2, AF-3 : Afi site 3, AF-4 : Afi site 4, Moy : Moyenne ; CV : Coefficient de Variation..

Tableau 4: Nitrates $\left(\mathrm{NO}_{3}^{-}\right)$, Nitrites $\left(\mathrm{NO}_{2}^{-}\right)$, Ammonium $\left(\mathrm{NH}_{4}^{+}\right)$et Orthophosphate mesurés dans les mares.

\begin{tabular}{|c|c|c|c|c|c|c|c|c|c|}
\hline \multirow[t]{2}{*}{ Mares } & \multirow[t]{2}{*}{ Paramètres } & \multicolumn{2}{|c|}{$\mathrm{NO}_{3}^{-}(\mathrm{mg} / \mathrm{L}$} & \multicolumn{2}{|c|}{$\mathrm{NO}_{2}^{-}(\mathrm{mg} / \mathrm{L})$} & \multicolumn{2}{|c|}{$\mathrm{NH}_{4}^{+}(\mathrm{mg} / \mathrm{L})$} & \multicolumn{2}{|c|}{$\mathrm{PO}_{4}{ }^{3-}(\mathrm{mg} / \mathrm{L})$} \\
\hline & & juillet & août & juillet & août & juillet & août & juillet & août \\
\hline \multirow{5}{*}{ Dindin } & DI-1 & $<0,5$ & $<0,5$ & $<0,025$ & $<0,025$ & $<0,05$ & 0,11 & $<0,2$ & $<0,2$ \\
\hline & DI-2 & $<0,5$ & $<0,5$ & $<0,025$ & $<0,025$ & $<0,05$ & 0,18 & $<0,2$ & $<0,2$ \\
\hline & Moy & - & - & - & - & & 0,145 & - & - \\
\hline & CV $(\%)$ & - & - & - & - & & 34,14 & - & - \\
\hline & LO-1 & $<0,5$ & $<0,5$ & $<0,025$ & $<0,025$ & $<0,05$ & 0,18 & $<0,2$ & $<0,2$ \\
\hline \multirow[t]{4}{*}{ Lotoè } & LO-2 & $<0,5$ & $<0,5$ & $<0,025$ & $<0,025$ & $<0,05$ & 0,11 & $<0,2$ & $<0,2$ \\
\hline & Moy & - & - & - & - & - & 0,145 & - & - \\
\hline & CV $(\%)$ & - & - & - & - & - & 34,14 & - & - \\
\hline & LA-1 & $<0,5$ & $<0,5$ & $<0,025$ & $<0,025$ & $<0,05$ & 0,09 & $<0,2$ & $<0,2$ \\
\hline \multirow[t]{5}{*}{ Lagoè } & LA-2 & $<0,5$ & $<0,5$ & $<0,025$ & $<0,025$ & $<0,05$ & 0,18 & $<0,2$ & $<0,2$ \\
\hline & Moy & - & - & - & - & - & 0,135 & - & - \\
\hline & CV $(\%)$ & - & - & - & - & - & 47,17 & - & - \\
\hline & AF-1 & $<0,5$ & $<0,5$ & $<0,025$ & $<0,025$ & $<0,05$ & 0,07 & $<0,2$ & $<0,2$ \\
\hline & AF-2 & $<0,5$ & $<0,5$ & $<0,025$ & $<0,025$ & $<0,05$ & 0,11 & $<0,2$ & $<0,2$ \\
\hline \multirow[t]{4}{*}{ Afi } & AF-3 & $<0,5$ & $<0,5$ & $<0,025$ & $<0,025$ & $<0,05$ & 0,11 & $<0,2$ & $<0,2$ \\
\hline & AF-4 & $<0,5$ & 0,53 & $<0,025$ & $<0,025$ & $<0,05$ & 0,07 & $<0,2$ & $<0,2$ \\
\hline & Moy & - & - & - & - & - & $\mathbf{0 , 0 9}$ & - & - \\
\hline & CV (\%) & - & - & - & - & - & 25,66 & - & - \\
\hline
\end{tabular}

DI-1 : Dindin site 1, DI-2 : Dindin site 2, LO-1 : Lotoè site 1, LO-2 : Lotoè site 2, LA-1 : Lagoè site 1, LA-2 : Lagoè site 2, AF-1 : Afi site 1, AF-2 : Afi site 2, AF-3 : Afi site 3, AF-4 : Afi site 4, Moy : Moyenne ; CV : Coefficient de Variation. 
Tableau 5: Transparence et profondeur mesurées dans les mares.

\begin{tabular}{llllll}
\hline Mares & Paramètres & \multicolumn{2}{l}{ Profondeur $(\mathbf{m})$} & \multicolumn{2}{l}{ Transparence (cm) } \\
\hline \multirow{3}{*}{ Dindin } & & juillet & août & juillet & août \\
& DI-1 & 1,95 & 1,9 & 70 & 60 \\
& DI-2 & 2 & 2 & 85 & 70 \\
& Moy & $\mathbf{1 , 9 7 5}$ & $\mathbf{1 , 9 5}$ & $\mathbf{7 7 , 5}$ & $\mathbf{6 5}$ \\
& CV $(\%)$ & $\mathbf{1 , 7 9}$ & $\mathbf{3 , 6 3}$ & $\mathbf{1 3 , 7}$ & $\mathbf{1 0 , 9}$ \\
& LO-1 & 2,1 & 2,1 & 110 & 95 \\
& LO-2 & 2,25 & 2,2 & 95 & 95 \\
& Moy & $\mathbf{2 , 1 7 5}$ & $\mathbf{2 , 1 5}$ & $\mathbf{1 0 3}$ & $\mathbf{9 5}$ \\
& CV $(\%)$ & $\mathbf{4 , 8 8}$ & $\mathbf{3 , 2 9}$ & $\mathbf{1 0 , 4}$ & $\mathbf{0}$ \\
& LA-1 & 2,5 & 2,4 & 120 & 115 \\
& LA-2 & 2,9 & 2,55 & 135 & 130 \\
& Moy & $\mathbf{2 , 7}$ & $\mathbf{2 , 4 8}$ & $\mathbf{1 2 8}$ & $\mathbf{1 2 3}$ \\
& CV $(\%)$ & $\mathbf{1 0 , 4 8}$ & $\mathbf{4 , 2 9}$ & $\mathbf{8 , 3 2}$ & $\mathbf{8 , 6 6}$ \\
& AF-1 & 2,2 & 2,15 & 75 & 55 \\
& AF-2 & 2,1 & 2,05 & 90 & 75 \\
& AF-3 & 2,5 & 2,45 & 105 & 95 \\
& AF-4 & 2,9 & 2,85 & 104 & 130 \\
& Moy & $\mathbf{2 , 4 2 5}$ & $\mathbf{2 , 3 8}$ & $\mathbf{9 3 , 5}$ & $\mathbf{8 8 , 8}$ \\
& CV $(\%)$ & $\mathbf{1 4 , 8 2}$ & $\mathbf{1 5 , 1}$ & $\mathbf{1 5 , 1}$ & $\mathbf{3 6}$ \\
\hline
\end{tabular}

DI-1 : Dindin site 1, DI-2 : Dindin site 2, LO-1 : Lotoè site 1, LO-2 : Lotoè site 2, LA-1 : Lagoè site 1, LA-2 : Lagoè site 2, AF-1 : Afi site 1, AF-2 : Afi site 2, AF-3 : Afi site 3, AF-4 : Afi site 4, Moy : Moyenne ; CV : Coefficient de Variation.

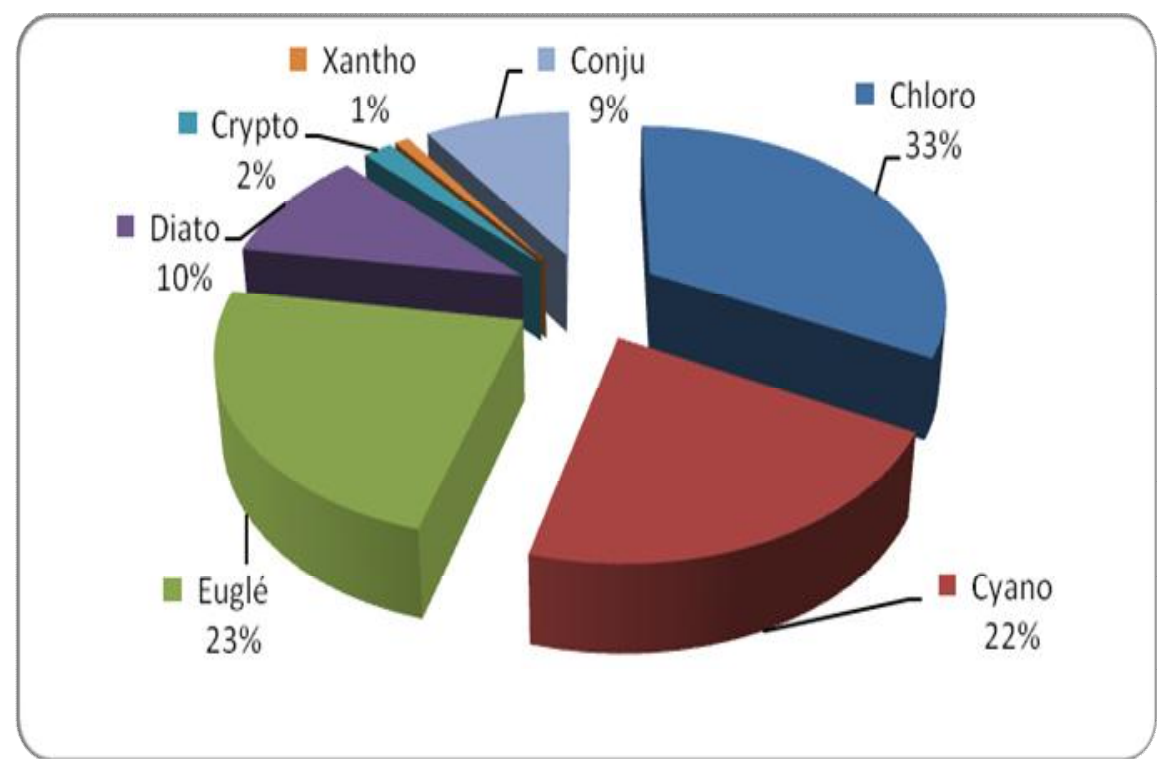

Figure 2: Composition qualitative du phytoplancton des mares. Crypto $=$ Cryptophycées, Chloro $=$ Chlorophycées, Euglé $=$ Euglénophycées, Cyano $=$ Cyanophycées, Diato $=$ Diatomophycées, Xantho $=$ Xanthophycées, Conju $=$ Conjugatophycées. 
Tableau 6: Densité des classes phytoplanctoniques des mares Dinidn, Lotoè et Lagoè.

\begin{tabular}{|c|c|c|c|c|c|c|}
\hline \multicolumn{7}{|c|}{ Mare Dindin } \\
\hline & & \multicolumn{2}{|c|}{ juillet } & \multicolumn{3}{|c|}{ août } \\
\hline Classes & S1 & S2 & Moy & S1 & S2 & Moy \\
\hline Chlorophycées $\left(10^{3}\right.$ ind/L) & 28 & 9 & $\mathbf{1 8 , 5} \pm 13,19$ & 8 & 17 & $\mathbf{1 2 , 5} \pm 6,60$ \\
\hline Conjugatophycées $\left(10^{3}\right.$ ind/L) & 6 & 10 & $\mathbf{8} \pm 2,82$ & 2 & 1 & $\mathbf{2} \pm 0,94$ \\
\hline Cryptophycées $\left(10^{3} \mathrm{ind} / \mathrm{L}\right)$ & 0 & 2 & $\mathbf{1} \pm 1,88$ & 0 & 9 & $\mathbf{4 , 5} \pm 6,60$ \\
\hline Cyanophycées $\left(10^{3} \mathrm{ind} / \mathrm{L}\right)$ & 9 & 6 & $\mathbf{7 , 5} \pm 1,88$ & 2 & 14 & $8 \pm 8,48$ \\
\hline Diatomophycées $\left(10^{3} \mathrm{ind} / \mathrm{L}\right)$ & 10 & 14 & $\mathbf{1 2} \pm 2,82$ & 0 & 8 & $4 \pm 5,65$ \\
\hline Xanthophycées (103 ind/L) & 1 & 0 & $\mathbf{0 , 5 0} \pm 0,94$ & 0 & 0 & $\mathbf{0}$ \\
\hline Euglenophycées $\left(10^{3} \mathrm{ind} / \mathrm{L}\right)$ & 0 & 100 & $\mathbf{5 0} \pm 70,71$ & 33 & 148 & $\mathbf{9 0 , 5} \pm 81,08$ \\
\hline \multicolumn{7}{|c|}{ Mare Lotoè } \\
\hline Chlorophycées $\left(10^{3}\right.$ ind/L) & 9 & 17 & $\mathbf{1 3} \pm 5,66$ & 0 & 9 & $4,5 \pm 6,60$ \\
\hline Conjugatophycées $\left(10^{3}\right.$ ind/L) & 4 & 0 & $\mathbf{2} \pm 2,83$ & 0 & 4 & $\mathbf{2} \pm 2,82$ \\
\hline Cryptophycées $\left(10^{3} \mathrm{ind} / \mathrm{L}\right)$ & 0 & 5 & $\mathbf{2 , 5} \pm 3,77$ & 93 & 6 & $\mathbf{4 9 , 5} \pm 1,88$ \\
\hline Cyanophycées $\left(10^{3}\right.$ ind/L) & 16 & 26 & $21 \pm 7,54$ & 4 & 9 & $\mathbf{6 , 5} \pm 3,77$ \\
\hline Diatomophycées $\left(10^{3} \mathrm{ind} / \mathrm{L}\right)$ & 16 & 0 & $\mathbf{8} \pm 11,31$ & 5 & 8 & $\mathbf{6 , 5} \pm 1,88$ \\
\hline Euglenophycées $\left(10^{3} \mathrm{ind} / \mathrm{L}\right)$ & 21 & 4 & $\mathbf{1 2 , 5} \pm 12,2$ & 82 & 44 & $\mathbf{6 3} \pm 27,3$ \\
\hline \multicolumn{7}{|c|}{ Mare Lagoè } \\
\hline Chlorophycées $\left(10^{3}\right.$ ind/L $)$ & 64 & 33 & $\mathbf{4 8 , 5} \pm 21,6$ & 50 & 78 & $\mathbf{6 4} \pm 19,8$ \\
\hline Conjugatophycées $\left(10^{3} \mathrm{ind} / \mathrm{L}\right)$ & 21 & 10 & $\mathbf{1 5 , 5} \pm 7,54$ & 8 & 12 & $\mathbf{1 0} \pm 2,8$ \\
\hline Cryptophycées $\left(10^{3} \mathrm{ind} / \mathrm{L}\right)$ & 45 & 30 & $\mathbf{3 7 , 5} \pm 10,37$ & 2 & 2 & $2 \pm 0$ \\
\hline Cyanophycées $\left(10^{3} \mathrm{ind} / \mathrm{L}\right)$ & 2066 & 1020 & $\mathbf{1 5 4 3} \pm 740,1$ & 512 & 520 & $\mathbf{5 1 6} \pm 5,65$ \\
\hline Diatomophycées $\left(10^{3} \mathrm{ind} / \mathrm{L}\right)$ & 29 & 13 & $\mathbf{2 1} \pm 11,3$ & 20 & 10 & $\mathbf{1 5} \pm 65,9$ \\
\hline Euglenophycées $\left(10^{3} \mathrm{ind} / \mathrm{L}\right)$ & 50 & 17 & $\mathbf{3 3 , 5} \pm 23,57$ & 37 & 144 & $\mathbf{9 0} \pm 75,4$ \\
\hline
\end{tabular}

Tableau 7: Densité des classes phytoplanctoniques de la mare Afi.

\begin{tabular}{|c|c|c|c|c|c|c|c|c|c|c|}
\hline \multicolumn{11}{|c|}{ Afi } \\
\hline & \multicolumn{7}{|c|}{ juillet } & \multicolumn{3}{|c|}{ août } \\
\hline Classes & S1 & S2 & S3 & S4 & Moy & S1 & S2 & S3 & S4 & Moy \\
\hline Chlorophycées $\left(10^{3}\right.$ ind/L) & 330 & 256 & 309 & 330 & $\mathbf{3 0 6 , 2} \pm 35,2$ & 70 & 426 & 490 & 149 & $\mathbf{2 8 3 , 7 5} \pm 205,5$ \\
\hline Conjugatophycées $\left(10^{3} \mathrm{ind} / \mathrm{L}\right)$ & 53 & 58 & 53 & 53 & $\mathbf{5 4 , 2} \pm 2,6$ & 6 & 224 & 149 & 64 & $\mathbf{1 1 0 , 7 5} \pm 95,4$ \\
\hline Cyanophycées $\left(10^{3}\right.$ ind $\left./ \mathrm{L}\right)$ & 3226 & 3200 & 3264 & 3226 & $3229 \pm 26,3$ & 461 & 4704 & 4661 & 3594 & $\mathbf{3 3 3 5} \pm 1996,4$ \\
\hline Diatomophycées $\left(10^{3} \mathrm{ind} / \mathrm{L}\right)$ & 96 & 96 & 96 & 96 & $\mathbf{9 6} \pm 0$ & 8 & 160 & 117 & 85 & $\mathbf{9 2 , 5} \pm 64,1$ \\
\hline Euglenophycées $\left(10^{3} \mathrm{ind} / \mathrm{L}\right)$ & 32 & 32 & 32 & 26 & $\mathbf{3 0 , 5} \pm 2,6$ & 24 & 149 & 74 & 245 & $\mathbf{1 2 3} \pm 96,2$ \\
\hline
\end{tabular}




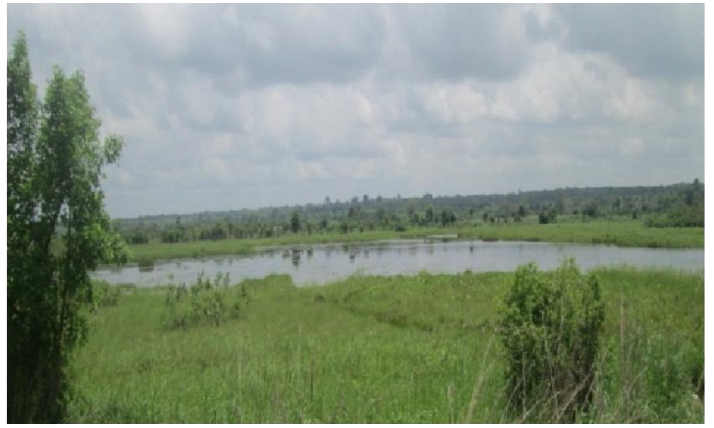

Photo 1: Mare Dindin

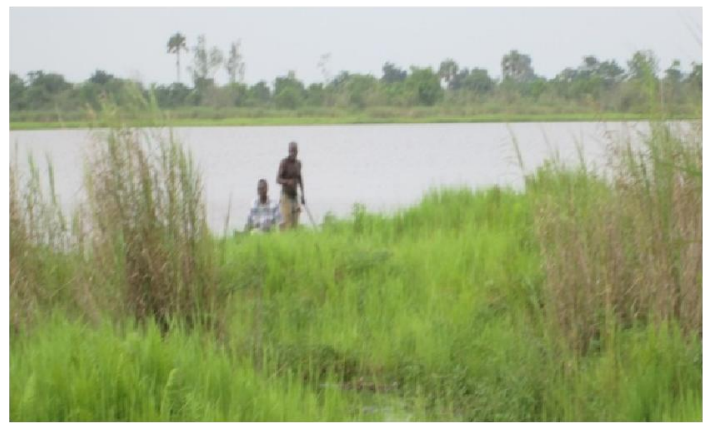

Photo 3: Mare Lagoè

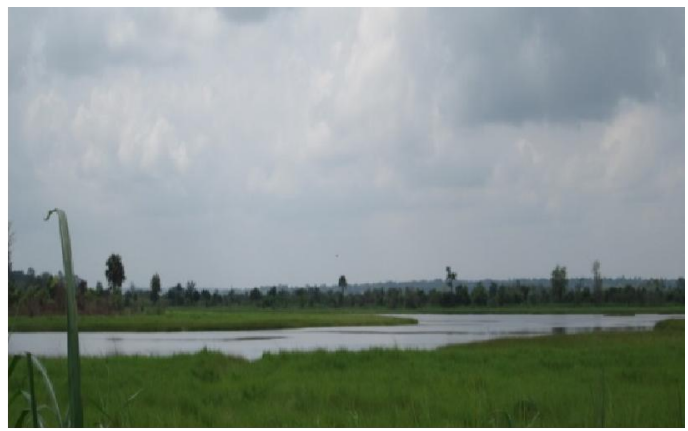

Photo 2 : Mare Lotoè

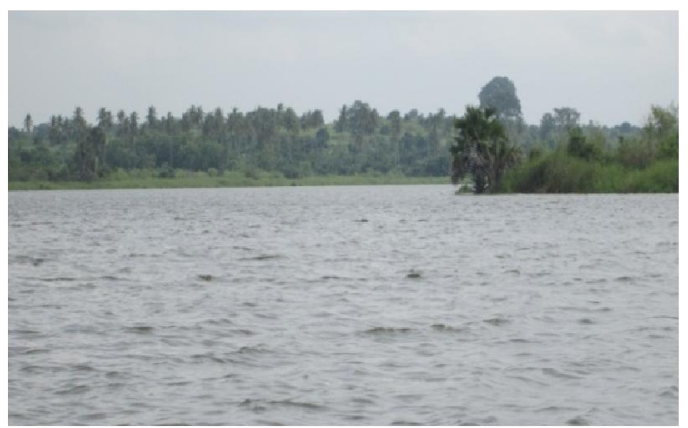

Photo 4: Mare Afi

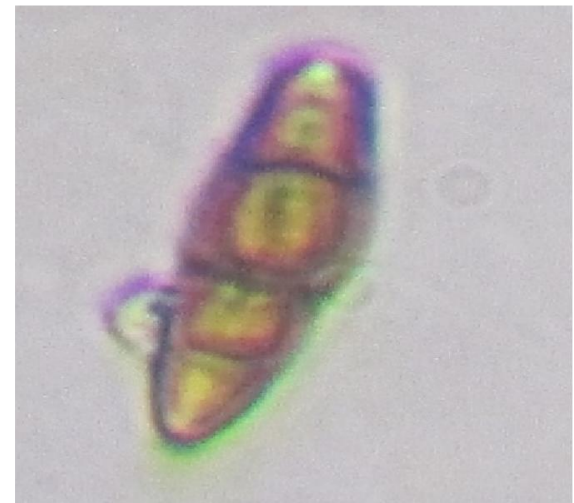

Tetracyclus rupestris (Chlorophycées)

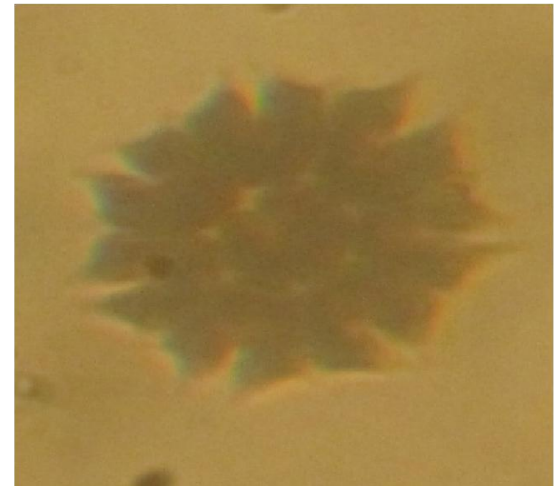

Pediastrum tetras (Chlorophycées)

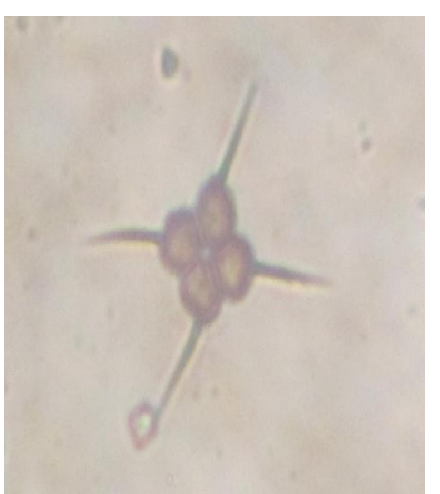

Tetrastrum heteracanthum (Chlorophycées) 


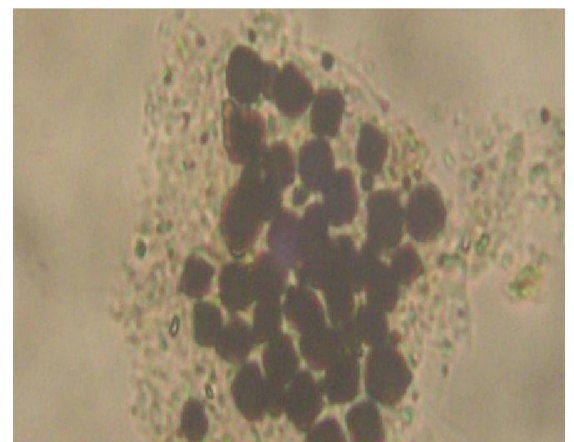

Microcystis aeruginosa (Cyanophycées)

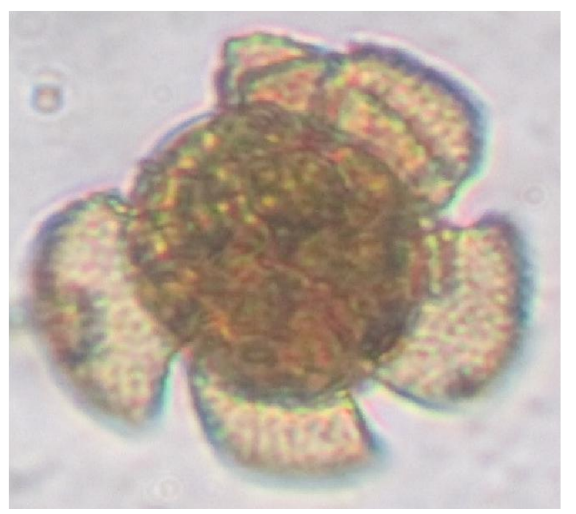

Trachelomonas volvocinopsis (Euglénophycées)

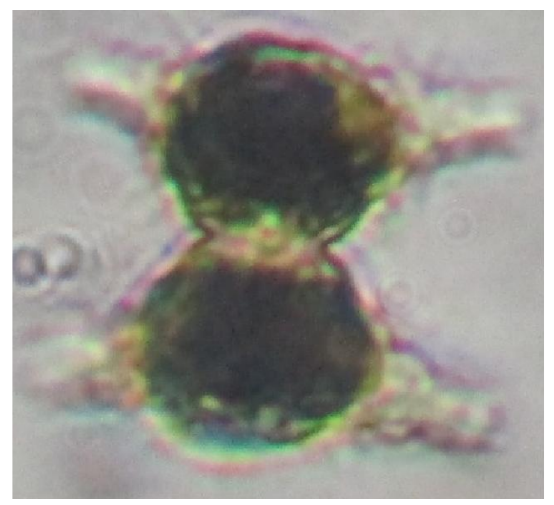

Staurastrum volans

(Conjugatophycées)

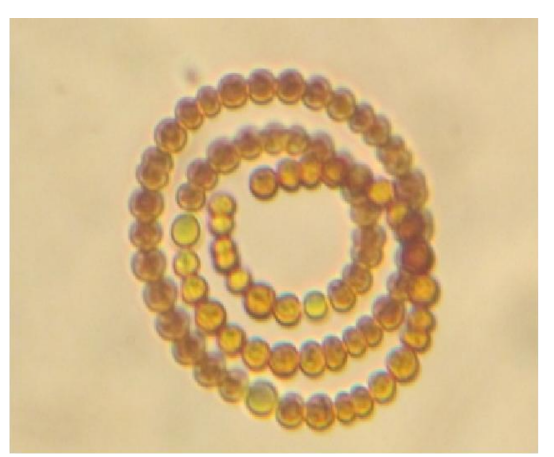

Anabaena spiroides (Cyanophycées)

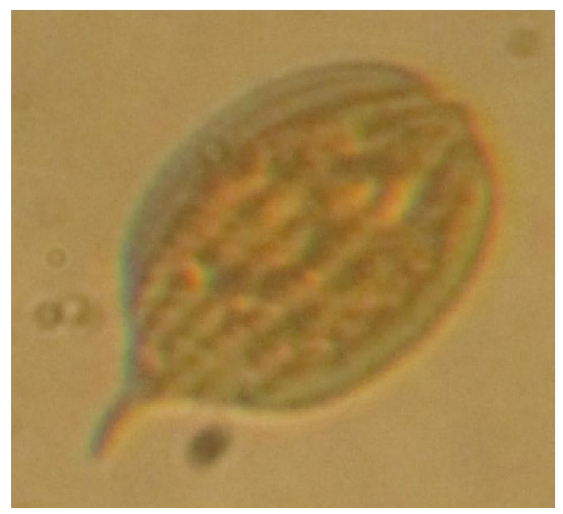

Phacus succicus (Euglénophycées)

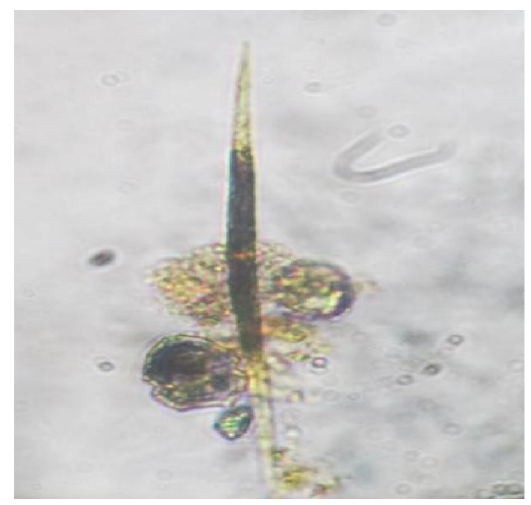

Closterium cornu

(Conjugatophycées)

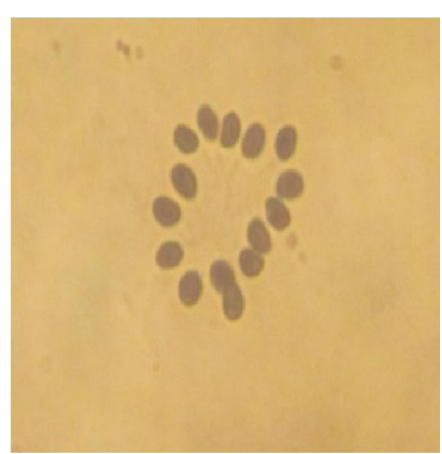

Coelomoron pusil

(Cyanophycées)

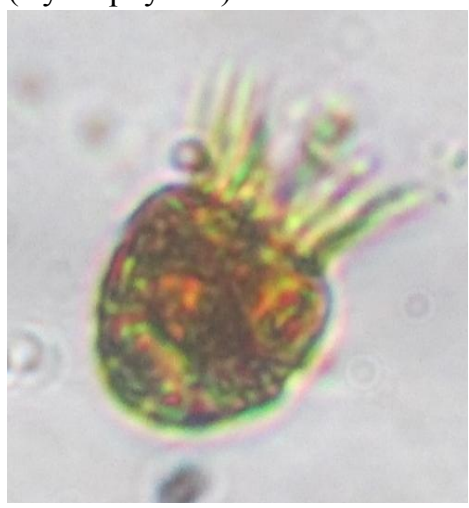

Trachelomonas armata (Euglénophycées)

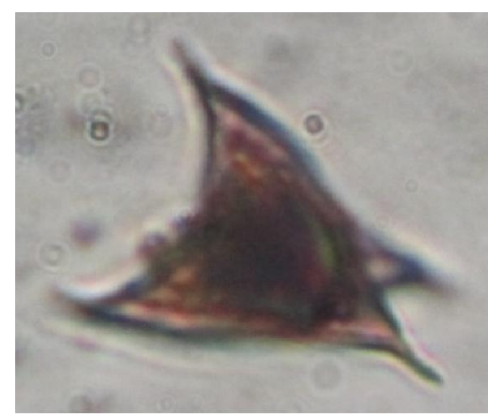

Tetraplektron torsum (Xanthophycées)

Planche 1 : Quelques espèces phytoplanctoniques identifiées. 


\section{DISCUSSION}

Des résultats de cette étude, nous pouvons dire que les eaux des mares (Dindin, lotoè, lagoè et afi) sont restées chaudes tout au long des deux mois d'étude (un minimum de $27,2^{\circ} \mathrm{C}$ à un maximum de $28,8^{\circ} \mathrm{C}$ ). Mais, les températures enregistrées sont plus basses en août. Cela serait dû à la fraîcheur du vent de mousson qui souffle en cette période dans la zone d'étude associée à la constitution géographique de ces mares avec leur forte végétation bordante. La température chaude de ces plans d'eau est comparable à celle du lac Taabo de la Côte d'Ivoire (Groga, 2012) et leurs valeurs basses en août sont comparables à celles du lac Zowla au Togo (Atanle et al., 2013). Ces valeurs de température enregistrées dans ces mares correspondent à celles souvent observées dans les milieux aquatiques à climat subéquatorial (Agadjihouèdé et al., 2016).

Selon cette relation existant entre la minéralisation et la conductivité électrique (Ahadjitsè, 1991), les eaux de la mare Afi $(408,5 \mu \mathrm{S} / \mathrm{cm}, \mathrm{CV}=0,14$ en juillet et 416,5 $\mu \mathrm{S} / \mathrm{cm}, \mathrm{CV}=0,31$ en août), Lagoè (404 $\mu \mathrm{S} / \mathrm{cm}, \mathrm{CV}=0$ en juillet et $414 \mu \mathrm{S} / \mathrm{cm}, \mathrm{CV}=$ 0 en août) et Lotoè $(388,5 \mu \mathrm{S} / \mathrm{cm}, \mathrm{CV}=1,64$ en juillet et $392,5 \mu \mathrm{S} / \mathrm{cm}, \mathrm{CV}=0,54$ en août) sont moyennement minéralisées. Par contre, l'eau de la mare Dindin est très minéralisée. Les valeurs de la conductivité de l'eau enregistrées dans ces mares sont inférieures aux valeurs enregistrées dans le lac Taabo en côte d'Ivoire (Groga, 2012) ainsi que dans les plans d'eau Azili, Hlan et Toho au Bénin (Houssou, 2011). La valeur élevée de la conductivité électrique de ces eaux serait due à la minéralisation de la grande quantité de matière organique provenant des débris de macrophytes morts (Atanle et al., 2012) ou d'un taux élevé de particules en suspension dans le lac (O.B.V, 2012). Selon CRELaurentides $(2009,2011)$, une conductivité supérieure à $125 \mu \mathrm{S} / \mathrm{cm}$ peut démontrer de l'influence des activités humaines via notamment l'apport de sels.
Pour ce qui est du pH, de la turbidité et de l'oxygène dissous, la mare Afi et Lagoè sont caractérisées par leurs valeurs élevées. Les valeurs du $\mathrm{pH}$ sont dans les normes de survie et d'évolution de la vie aquatique. Elles suivent l'échelle de l'I.B.G.E (2005) pour lequel un $\mathrm{pH}$ naturel situé entre 6,5 et 8,5 caractérise les eaux où la vie se développe de manière optimale. Les limites à l'origine des premiers dommages biologiques sont entre 5,5 et 6,5 tranches dans lesquelles les espèces les plus intolérantes disparaissent (Dupont, 2004). S'agissant de la turbidité, c'est un paramètre qui permet d'évaluer la quantité de matières en suspension dans l'eau. La valeur élevée de la turbidité de ces mares Afi et Lagoè peut être due à la remise en suspension des particules déposées au fond par les fortes agitations de l'eau occasionnée par le vent de la mousson et la mise en suspension de la forte quantité de matière organique présente dans l'eau. Ceci est en lien avec les valeurs faibles de la transparence obtenue dans ces mares et des valeurs élevées du $\mathrm{pH}$; car les substances humiques qui ont occasionné l'augmentation de la turbidité ne sont pas encore transformées en acide humique. Des observations semblables ont été faites dans le lac Taabo de la côte d'Ivoire (Groga, 2012) et dans le lac Zowla au Togo (Atanle et al., 2013).

Les teneurs d'oxygène dissous enregistrées à Afi et Lagoè pourraient amener à dire que ces mares appartiennent à la catégorie des lacs à eutrophisation avancée ; $\mathrm{O}_{2}>6 \mathrm{mg} / \mathrm{L}$ (I.B.G.E, 2005) au sein desquels le taux élevé de photosynthèse dû au bloom algal libère de grandes quantités d'oxygène. Mais, en réalité, c'est la consommation de l'oxygène dissous lors de la dégradation microbienne de la matière organique qui est néfaste pour le milieu eutrophié. Ainsi en se référant à la température de l'eau de ces mares et à la pression atmosphérique standard de $101,3 \mathrm{KPa}$, la concentration saturée théorique 
calculée de l'oxygène dissous est de 7,68 $\mathrm{mg} / \mathrm{L}$ pour Afi et de $7,76 \mathrm{mg} / \mathrm{L}$ pour Lagoè et montre que ces mares sont à la limite de l'eutrophisation. En effet, à une pression atmosphérique standard de $101.3 \mathrm{KPa}$, un plan d'eau au niveau de la mer aurait une concentration saturée de 7,$96 ; 7,82$ et 7,68 $\mathrm{mg} / \mathrm{L}$ aux températures de 27,28 et $29{ }^{\circ} \mathrm{C}$ respectivement et un plan d'eau qui présente des concentrations supérieures est influencé par une activité photosynthétique importante. Ces résultats sont comparables à ceux obtenus sur le lac Toho au Bénin où la valeur de l'oxygène dissous est supérieure à la norme (Houssou, 2011). Quant à l'oxydabilité au $\mathrm{KMnO}_{4}$, selon Atanle et al. (2013), l'augmentation du taux de matières organiques dans une eau pourrait être due à la décomposition des macrophytes présents dans cette eau. Ce qui expliquerait les valeurs élevées de l'oxydabilité au $\mathrm{KMnO}_{4}$. Ces résultats sont aussi comparables à ceux de Atanle et al. (2012) qui oscille entre 6 et 12 $\mathrm{mg} / \mathrm{L}$.

Les mares Lagoè et Lotoè se distinguent par de très fortes valeurs de la profondeur et de la transparence au cours de cette étude. Ces valeurs de transparence seraient dues au développement des communautés zooplanctoniques. Ces derniers contribuent en effet par le broutage du phytoplancton, à la clarification des eaux dans les lacs réservoirs. Ces résultats ont été confirmés par ceux de Houssou (2011) au niveau du lac Hlan où ces valeurs sont supérieures à celles des autres lacs étudiés. Par contre, les valeurs faibles dans les autres mares seraient liées aux vents humides dominants en cette période et aussi à l'état d'eutrophisation du plan d'eau.

La présente étude a permis de recenser au total 107 espèces phytoplanctoniques au cours des deux mois d'étude (juillet et août).
Ce nombre est inférieur respectivement à celui rapporté par les travaux de Bandjé (2010) dans les écosystèmes du Sud-Ouest du Togo (203) et de Issifou et al. (2014) sur le système lagunaire de Lomé (117). Par contre, ce nombre est supérieur à celui obtenu par Atanle et al. (2012) dans le lac de Zowla (68). Dans l'ensemble des mares et au cours des deux mois d'étude, ce sont les Chlorophycées qui sont plus diversifiées avec $33 \%$ du peuplement phytoplanctonique. Tel n'est pas le cas dans les travaux de Atanle et al. (2012) où c'est la classe des Diatomophycées qui a une richesse spécifique élevée au cours de ses deux mois d'étude (juillet et août). Par contre, ces résultats sont semblables à ceux de $\mathrm{Ba}$ (2006) après la construction du barrage antisel de Diama sur le fleuve Sénégal où il est noté une forte dominance des Chlorophycées; car le fonctionnement du lac est devenu totalement contrôlé, les intrusions salines de l'eau de mer sont arrêtées et les eaux sont douces en permanence. Donc, dans les eaux douces, ce sont les Chlorophycées qui ont une plus grande richesse spécifique.

Sur la mare Afi et Lagoé, cette étude a permis de recenser respectivement 62 et 65 espèces dans chacune, tout au long de cette étude. A Dindin et Lotoé, respectivement 46 et 37 espèces étaient identifiées. Ces richesses spécifiques sont faibles par rapport à celles reportées pour certains lacs de la zone comme le lac Guiers au Sénégal (111 espèces) (Ngansoumana, 2006) etc. Par contre, ces mares sont plus riches au niveau spécifique que le lac Azili, Hlan et Toho du Bénin où respectivement 55,40 et 27 espèces ont été déterminées (Houssou, 2011). La forte diversité en espèces permettrait une plus grande stabilité du fonctionnement de l'écosystème face aux perturbations environnementales. Il s'avère ici que les trois plans d'eau sont pauvres du point de vue 
richesse spécifique, mais une stabilité est notée entre les mois d'étude. Dans les mares Lagoè et Afi, il est noté la dominance des Cyanophycées avec une forte composante cyanobactérienne. Les individus concernés sont du genre Anabaena, Microcystis, Anabaenopsis, Merismopedia, Chroococcus, Spirulina, Cylindrospermopsis, Aphanothece et Wollea. Dans la mare Dindin, une dominance des Euglénophycées est notée avec des genres tels que Phacus, Euglena et Trachelomonas. La mare Lotoè quant à elle est à dominance Cyanophytes en juillet et Euglénophytes en août.

Il faut noter une corrélation entre les paramètres physico-chimiques et la diversité phytoplanctonique. Les Euglénophycées et les Xanthophycées se développent mieux dans les milieux riches en $\mathrm{NH}_{4}^{+}, \mathrm{KMnO}_{4}$ et à conductivité élevées. Ceci est le cas de la mare Dindin caractérisée par une conductivité, un taux de $\mathrm{NH}_{4}^{+}$et $\mathrm{KMnO}_{4}$ élevée en août où les Euglénophycées y dominent $(72,96 \%$ en juillet $69,44 \%$ en août). Ces résultats sont similaires à ceux obtenues par Atanle et al. (2012) dans ses travaux sur le lac Zowla.

Les Cyanophycées, les Chlorophycées, les Conjugatophycées et les Diatomophycées ont une forte affinité pour les milieux à $\mathrm{pH}$, turbidité et oxygène dissous élevés. C'est le cas de la mare Afi et Lagoè caractérisées par une turbidité, un taux d'oxygène dissous et un $\mathrm{pH}$ élevé et qui est dominé par les Cyanophycées (86,88\% en juillet et $83,90 \%$ en août) suivi des Chlorophycées et celle de la mare Lagoè $(90,70 \%$ en juillet et $74,44 \%$ en août). Ces résultats sont en adéquation avec les travaux de Sanaa (2006) selon qui les Cyanophycées expriment leur dominance prodigieuse dans les eaux à forte turbidité. Cette forte présence des Cyanophycées peut être expliquée par leur capacité à croître sous de faibles intensités lumineuses associées à de grandes turbidités caractéristiques de ces mares (Chorus et Bartram, 1999).

\section{Conclusion}

Les résultats de cette étude ont montré qu'en général les mares Afi et Lagoè sont des mares caractérisées par une turbidité, un $\mathrm{pH}$, un taux d'oxygène dissous élevés avec une dominance des Cyanophycées. Lotoè est plus particulière par sa profondeur et sa transparence. Quant à Dindin, elle se différencie des autres par une conductivité électrique et un taux d'oxydabilité au $\mathrm{KM}_{\mathrm{n}} \mathrm{O}_{4}$ élevées et une dominance des Euglénophycées. Le taux élevé d'oxygène dissous des mares Afi et Lagoè fait de ces mares des milieux eutrophes.

\section{CONFLIT D'INTERETS}

Les auteurs déclarent qu'ils n'ont aucun conflit d'intérêts.

\section{CONTRIBUTIONS DES AUTEURS}

La collecte des données est réalisée par DLA. Les traitements des données et la rédaction du manuscrit étaient faits par DLA et HA. Tout le travail est supervisé par KK et APL responsables des laboratoires où les travaux ont été conduits.

\section{REMERCIEMENTS}

Les auteurs tiennent à remercier tous les lecteurs anonymes pour leur contribution à la qualité scientifique de ce manuscrit.

\section{REFERENCES}

AFNOR. 1997. Qualité de l'Eau (Tome 2), Méthodes d'Analyse 1 : Analyse Organoleptique, Mesures Physicochimiques, Paramètres Globaux, Composés Organiques ( ${ }^{\mathrm{e}}$ édn). AFNOR: Paris.

Agadjihouèdé $\mathrm{H}$, Bonou AC, Ahouansou Montcho $\mathrm{S}$, Montchowui $\mathrm{E}$, Lalèyè $\mathrm{P}$. 
2016. Composition et abondance du peuplement zooplanctonique des étangs piscicoles du périmètre rizicole de Malanville (Nord Bénin). Bulletin de la Recherche Agronomique du BéninNuméro spécial Agronomie, Société, Environnement \& Sécurité Alimentaire: 70-78. http://www.slire.net

Ahadjitse NK. 1991. Contribution à l'étude chimique des eaux souterraines de la région de Kévé. Mémoire de Technicien Supérieur en Génie Sanitaire, p. 74.

Atanle K, Bawa ML, Kokou K, DjaneyeBoundjou G. 2012. Caractérisation physico-chimique et diversité phytoplanctonique des eaux du lac de Zowla (Lac Boko), Togo. Int. J. Biol. Chem. Sci., 6(1): 543-558. DOI: http://ajol.info/index.php/ijbcs

Atanle K, Bawa ML, Kokou K, Djaneyeboundjou G, Edorh MT. 2013. Distribution saisonnière du phytoplancton en fonction des caractéristiques physico-chimiques du lac de zowla (lac boko) dans le sud- est du togo, Journal of Applied Biosciences, 64: 4847-4857.

Azam F, Malfatti F. 2007. Microbial Structuring of marine ecosystems. Nature Reviews Microbiology, 5: 782791.

Ba N. 2006. La communauté phytoplanctonique du lac de Guiers (Sénégal): Types d'associations fonctionnelles et approches expérimentales des facteurs de régulation. Thèse de Doctorat du $3^{\mathrm{e}}$ Cycle, Université Cheikh Anta Diop de Dakar (Sénégal), p. 130.

Bandjé A. 2010. Diversité et dynamique du phytoplancton dans les écosystèmes aquatiques du sud-ouest du Togo. Mémoire DEA-FDS/UL, p. 46.
Barnabé G. 1991. Bases Biologiques et Ecologiques de l'Aquaculture. Edition Lavoisier. TEC \& DOC. : Paris ; 489.

Bourrelly P. 1968. Les Algues Jaunes et Brunes (Tome II). Ed Boubée : Paris, p 438.

Bourrelly P. 1970. Les Algues Bleues et Rouges (Tome I). Ed Boubée: Paris; 511.

Bourrelly P. 1972. Les Algues Vertes (Tome III). Ed Boubée: Paris ; 572.

Chidi I. 1999. Profil Environnemental $d u$ Littoral du Togo. Presses de l'Université du Bénin : Bénin ; 403.

Conseil Régional de l'Environnement des Laurentides, 2011. Rapport d'interprétation des résultats 2009-2010. Lac des Becs-Scie, Municipalité de Saint-Sauveur. Suivi complémentaire de la qualité de l'eau du programme Bleu Laurentides, p 24.

Dupont J. 2004. La problématique des lacs acides au Québec. Direction du suivi de l'état de l'environnement, Ministère de l'Environnement, Envirodoq no ENV/2004/0151, collection no QE/145, p 18.

Edorh T, Blivi A, Bandjé A, Abotsi K. 2008. Présence d'algues toxiques dans les eaux marines et saumâtres du littoral togolais. Annales des Sciences Agronomiques du Bénin, 10(2): 165-177.

Groga N. 2012. Structure, fonctionnement et dynamique du phytoplancton dans le lac de Taabo (Côte d'Ivoire), Thèse de doctorat Université de Toulouse, 224 p.

Houssou MA. 2011. Variabilité saisonnière des communautés planctoniques (Phyto et zooplancton) des lacs Azili, Hlan et Toho. Mémoire de l'Université d'Abomey Calavi en vue d'obtention d'un Master; option Pêche et Aquaculture, 114 p. 
IBGE, 2005. Qualité physico-chimique et chimique des eaux de surface. Observatoire des Données de l'Environnement. Fiche de donnés, IBGE, $16 \mathrm{p}$.

Issifou L, Atanle K, Radji R, Lawson HL, Adjonou K, Edorh MT, Kokutse AD, Attoh-Mensah A, Kokou K. 2014. Checklist of tropical algae of Togo in the Guinean Gulf of West-Africa. Scientific Research and Essays, 9(22): 932-958. DOI: http://www.academicjournals.org/ SRE

Jiang JG, Shen YF. 2006. Estimation of the natural purification rate of a eutrophic lake after pollutant removal. Ecological Engineering, 28: 166-173.

Komarek J, Anagnostidis K. 1999. Susswasserfloravon Mitteleuropa 19/1. Cyanoprokaryota 2. Teil: Oscillatoriales. Elsevier Spektrum Akademischer Verlag éd., Elsevier: München; 548.
Lavoie I, Hamilton PB, Campeau S, Grenier M, Dillon P J. 2008. Guide d'Identification des Diatomées des rivières de l'Est du Canada. Presse de l'Université du Québec : Québec ; 241.

MERF. 2002. Monographie nationale sur la diversité biologique du Togo. MERF, $171 \mathrm{p}$.

Ngansoumana BA. 2006. La communauté phytoplanctonique du lac de Guiers (Sénégal) : types d'associations fonctionnelles et approches expérimentales des facteurs de régulation. Thèse de Doctorat de $3 \mathrm{e}$ Cycle de 1'Université Cheikh Anta Diop de Dakar (Sénégal). 155 p.

OBV, 2012. Bathymétrie et état trophique du lac Echo (Québec). Online, 18 p.

Tuzin D, Mason A. 1996. La clarification des eaux dans les lacs réservoirs, 56p. 